\title{
Motivación de los estudiantes de la Facultad de Ciencias de la Educación (Universidad deSevilla) hacia la práctica de actividad físico-deportiva Motivation of Students of Faculty of Education (University of Seville) to physical activity practice
}

\author{
Carolina Castañeda Vázquez y $\mathrm{M}^{\mathrm{a}}$ del Carmen Campos Mesa \\ Universidad de Sevilla
}

\begin{abstract}
Resumen: con esta investigación se pretende conocer las motivaciones de los estudiantes de la Facultad de Ciencias de la Educación de la Universidad de Sevilla hacia la actividad físico-deportiva. La muestra (N=409) está compuesta por estudiantes de esta Facultad ( $\pm 4.8 \%$; $95 \%$ CI) y para la obtención de datos se aplicó un cuestionario estructurado en 5 bloques, del cual se han seleccionado algunos ítems para esta investigación. Dicho instrumento fue validado por varios expertos en la materia y se han realizado diversas pruebas estadísticas con el paquete SPSS V.15, calculándose la fiabilidad del instrumento a través del coeficiente Alfa de Cronbach (.78) y realizándose análisis factoriales en las preguntas relativas a esta investigación. Los datos obtenidos revelan que los principales motivos de práctica están relacionados con la mejora de la salud y la estética, el disfrute y las relaciones sociales, mientras que los motivos aducidos para no haber practicado nunca o haber dejado de hacerlo, son muy semejantes y se centran en la disponibilidad temporal, el interés hacia la AFD y la oferta de práctica.
\end{abstract}

Palabras clave; Motivación, estudiantes universitarios, actividad física, deporte.

Abstract: the aim of this research is to study students' motivation for physical activity and sport in Faculty of Education of University of Seville. The sample $(\mathrm{N}=409)$ is constituted for students from this Faculty $( \pm 4.8 \% ; 95 \% \mathrm{CI})$ and a specific questionnaire, built to that effect and structured in five sections, was used to obtain dates. This instrument was validated by different experts on this area of studies, statistic tests was done to check its reliability (Alpha Cronbach: .78) and factor analysis has been done in certain items, using SPSS V.15. The main results showed that the main motives to physical activity practice are related to be in good health and aesthetics, enjoyment and social relations, meanwhile, the main motives to have not ever practice physical activity or have given it up, are both related to time availability or lack of time, interest to physical activity and sport offer.

Key words: Motivation, University students, physical activity, sport.

\section{Introducción}

La práctica de actividad físico-deportiva (AFD) regular y adecuada conlleva numerosos beneficios para la salud de los sujetos, los cuales han quedado ampliamente demostrados y han sido muy difundidos en los últimos años (Darren, Crystal \& Shannon, 2006; Castillo \& Pablos, 2007; Pìeron, Ruíz \& García, 2009). Aunque sigue existiendo un porcentaje amplio de la población que continua siendo inactivo, el número de personas que practican AFD evoluciona lenta pero positivamente. Así, en la última encuesta sobre hábitos deportivos en España (CSD, 2010), se ha observado un aumento en la práctica por parte de la población de entre 15 y 75 años, de un $37 \%$ en el año 2000, al $43 \%$ en el 2010; de manera que actualmente, alrededor de 16 millones de españoles mayores de 14 años practican deporte en nuestro país. Así mismo, también se ha confirmado un auge en las prácticas orientadas hacia la diversión y el pasatiempo, el mantenimiento de la salud (García, 2006) o hacia la mejora estética (Martínez-López, 2003), pues ya en 2005 , el $70 \%$ de la población activa, realizaba AFD sin preocuparse por competir (García, 2006).

Sin embargo, el nivel de práctica de AFD disminuye a medida que aumenta la edad (Trost, Kerr, Ward \& Rate, 2001; García, 2006; Van der Host, Paw, Twisk \& Van Mechelen, 2007; Ruiz, García \& Pieron, 2009;), de forma que las mayores tasas de práctica que se registran en la etapa infantil comienzan a decaer con la adolescencia, continuando ese descenso al llegar a la edad universitaria (Sánchez-Barrera, Pérez \& Godoy, 1995; García, 2001; López \& González, 2001; Pavón \& Moreno, 2006). Y es que la universidad puede suponer un cambio del estilo de vida para el alumnado, donde suelen producirse cambios de residencia, tienen más obligaciones, son necesarias más horas de estudio y se dispone por tanto de menos tiempo libre para la práctica. Así mismo, los estudiantes cuentan con una amplia oferta para ocupar ese tiempo de ocio, pues además de las actividades propiamente deportivas y en muchas ocasiones, prefieren ver la televisión, pasar tiempo con la fami-

Fecha recepción: 03-11-11- Fecha envío revisores: 03-11-11 - Fecha de aceptación: 07-12-11 Correspondencia: Carolina Castañeda Vázquez

Calle Pirotecnia, s/n.

41013. Sevilla

E-mail: carolinacv@us.es lia o salir con los amigos, en lugar de practicar algún deporte o AFD (García, 2001, 2006; Castillo, Giménez \& Sáenz-López, 2009). Según el Modelo del Deporte Universitario Español (CSD, 2006), el 64\% de los universitarios españoles realiza alguna $\mathrm{AFD}$, aunque ese porcentaje puede oscilar, habitualmente entre el $40 \%$ y el $60 \%$, en función de la universidad en cuestión (Palau, Ponseti \& Borrás, 2001; Sanz, 2005; Pavón \& Moreno, 2006; Castillo \& Sáenz-López, 2008).

Si consideramos la motivación hacia la AFD como el producto de un conjunto de variables de diversa índole (sociales, ambientales e individuales) que determinan aspectos de la práctica como la elección de una o varias actividades, la frecuencia, la intensidad, la persistencia en la actividad e incluso el rendimiento (Escarti \& Cervelló, 1994), el estudio de la misma puede ser un factor clave para favorecer la iniciación a la práctica, la adherencia a la misma y evitar el abandono, pues permite conocer por qué los sujetos eligen una actividad y los factores determinantes de la permanencia o abandono en esa elección (Moreno, Cervelló \& Martínez, 2007). De este modo, si desde las instituciones universitarias se pretende, tal y como se expone en la Ley Orgánica 6/2001, de 21 de diciembre, de Universidades y en la Ley Orgánica 4/2007, de 12 de abril, favorecer y promocionar la práctica de AFD como parte de la formación del alumnado, será interesante conocer las motivaciones que llevan a los estudiantes a practicar, abandonar o no haber realizado nunca AFD.

Por todo ello, el objetivo principal de esta investigación es conocer las motivaciones relacionadas con la práctica de AFD de los estudiantes de la Facultad de Ciencias de la Educación de la Universidad de Sevilla y, de manera más concreta, detectar los principales motivos que inducen al alumnado a practicar AFD, abandonar dicha práctica o no haberla realizado nunca., así como indagar sobre las razones por las que los alumnos que abandonaron la práctica de AFD estarían dispuestos a retomarla en un futuro cercano.

\section{Método}

La presente investigación se encuadra dentro de un estudio más amplio a través del que se pretenden conocer los hábitos de práctica de AFD de los estudiantes de la Facultad de Ciencias de la Educación de la Universidad de Sevilla. 
La muestra estáformada por 409 estudiantes, 184 hombres (44.8\%) y 226 mujeres (55.1\%), de la Facultad de Ciencias de la Educación de la Universidad de Sevilla $( \pm 4.8 \%$; $95 \% \mathrm{CI})$. En ésta se encuentran representadas todas las titulaciones de la Facultad de manera proporcional al número de alumnos matriculados en cada una de ellas, para no sesgar dicha muestra, ya que en este centro se imparten diferentes titulaciones de Educación Física.

\begin{tabular}{lcc}
\multicolumn{3}{c}{ Tabi a 1; Muestra de la investigación } \\
\hline Titul ación & Frecuencia & Porcent aje \\
\hline Grado CC AFD & 17 & $4.1 \%$ \\
Grado Pedago gía & 15 & $3.6 \%$ \\
Licenciatura CCAFD & 73 & $17.8 \%$ \\
Licenciatu ra en Pedagogía & 45 & $10.9 \%$ \\
Licenciatura en Psicopedagogía & 15 & $3.6 \%$ \\
Mag isterio Edu cación Es pecial & 36 & $8.7 \%$ \\
Mag isterio Edu cación Física & 98 & $23.9 \%$ \\
Magi isterio Edu cación Infantil & 13 & $3.1 \%$ \\
Mag isterio Edu cación Mu sical & 49 & $11.9 \%$ \\
Mag isterio Edu cación Primaria & 28 & $6.8 \%$ \\
Mag isterio Lengua Extranjera & 20 & $4.8 \%$ \\
\hline Total & 409 & $100 \%$
\end{tabular}

La media de edad de los encuestados es de 21.6 años $( \pm 4.26)$, encontrándose los mayores porcentajes en el intervalo de hasta 20 años (49\%).

Para obtener los datos se utilizó un cuestionario estructurado en cinco bloques diferenciados de preguntas del que se han seleccionado los siguientes ítems para la presente investigación:

P.13. ¿Te gustaría volver a practicar actividad físico-deportiva?

P.14. Valora las razones, en función de su importancia, que te ayudarían a retomar la práctica de actividades físico-deportivas.

P.15. Valora los motivos, en función de su importancia, por los que abandonaste la práctica de actividad físico-deportiva.

P.16. Valora los motivos, en función de su importancia, por los que nunca has practicado actividad físico-deportiva.

P.23. Valora los motivos, en función de su importancia, por los que realizas actividad físico-deportiva

Dicho cuestionario fue validado en primer lugar por varios expertos en la materia y se calculó el coeficiente de fiabilidad Alpha de Cronbach para determinar la consistencia interna del instrumento, obteniéndose una puntuación media de .78 puntos, que según Lowenthal (2001) y McMillan (2008) puede considerarse aceptable.

Se realizó un análisis factorial en las preguntas número $14,15,16$ y 23 mediante laextracción de componentes principales y rotación Varimax con Kaiser-Meyer-Olkin. Cada variable fue incluida en un solo factor, atendiendo a su carga factorial, estableciendo valores de ,30 como mínimo criterio de saturación (Ferrando \& Anguiano-Carrasco, 2010), que-

\begin{tabular}{|c|c|}
\hline \multicolumn{2}{|c|}{ Agrupación de ítems en la preg unta 14} \\
\hline Factores resul tantes & Variables \\
\hline $\begin{array}{l}\text { Factor 1: Motivos relacio nados con factores ambientales de la } \\
\text { práctica(recursos materiales y pers onales) }\end{array}$ & \begin{tabular}{|l|} 
Buenas instalaciones \\
Instalaciones cercanas \\
Monitores bien preparados
\end{tabular} \\
\hline $\begin{array}{l}\text { Factor 2: Motivos relacio nados con las características de la } \\
\text { práctica }\end{array}$ & $\begin{array}{l}\text { Practicar con amigos/famil iares } \\
\text { Actividades atractivas } \\
\text { Actividades más económicas }\end{array}$ \\
\hline $\begin{array}{l}\text { Factor 3: Motivos relacio nados con la dis poni bi lida d } \\
\text { temporal }\end{array}$ & $\begin{array}{l}\text { Tiempo organizado para estudiar } \\
\text { Horario de clases compatible }\end{array}$ \\
\hline \multicolumn{2}{|c|}{$\begin{array}{c}\text { Tabla 3; Factores resultantes para los motivos de abandono de la práctica de AFD } \\
\text { Agrupación de ítems en la preg unta } 15\end{array}$} \\
\hline Factor 1: Motivos de relación social & \begin{tabular}{|l|} 
No les enseñar on en la escuela \\
Sus amigos no practicaban \\
Sus padres no se lo inculcaron \\
Nadie le apoyó para iniciarse
\end{tabular} \\
\hline Factor 2: Motivos relacio nados con la oferta de práctica & $\begin{array}{l}\text { Falta de in stal aciones cercanas } \\
\text { Falta de in stal aciones adecuadas } \\
\text { No o fentaban actividades que les gustasen }\end{array}$ \\
\hline Factor 3: Motivos de relacionados con el interés hacia la AFD & $\begin{array}{l}\text { Falta de aptitu des para el deporte } \\
\text { No les gusta } \\
\text { Pereza y/o desgana }\end{array}$ \\
\hline $\begin{array}{l}\text { Factor 4: Motivos relacio nado s con la dis poni bi lida d } \\
\text { temporal }\end{array}$ & $\begin{array}{l}\text { Falta de tiempo } \\
\text { Salir cansado de las clases/trabajo }\end{array}$ \\
\hline Factor 5: Motivos económicos & Motivos eco nómicos \\
\hline
\end{tabular}

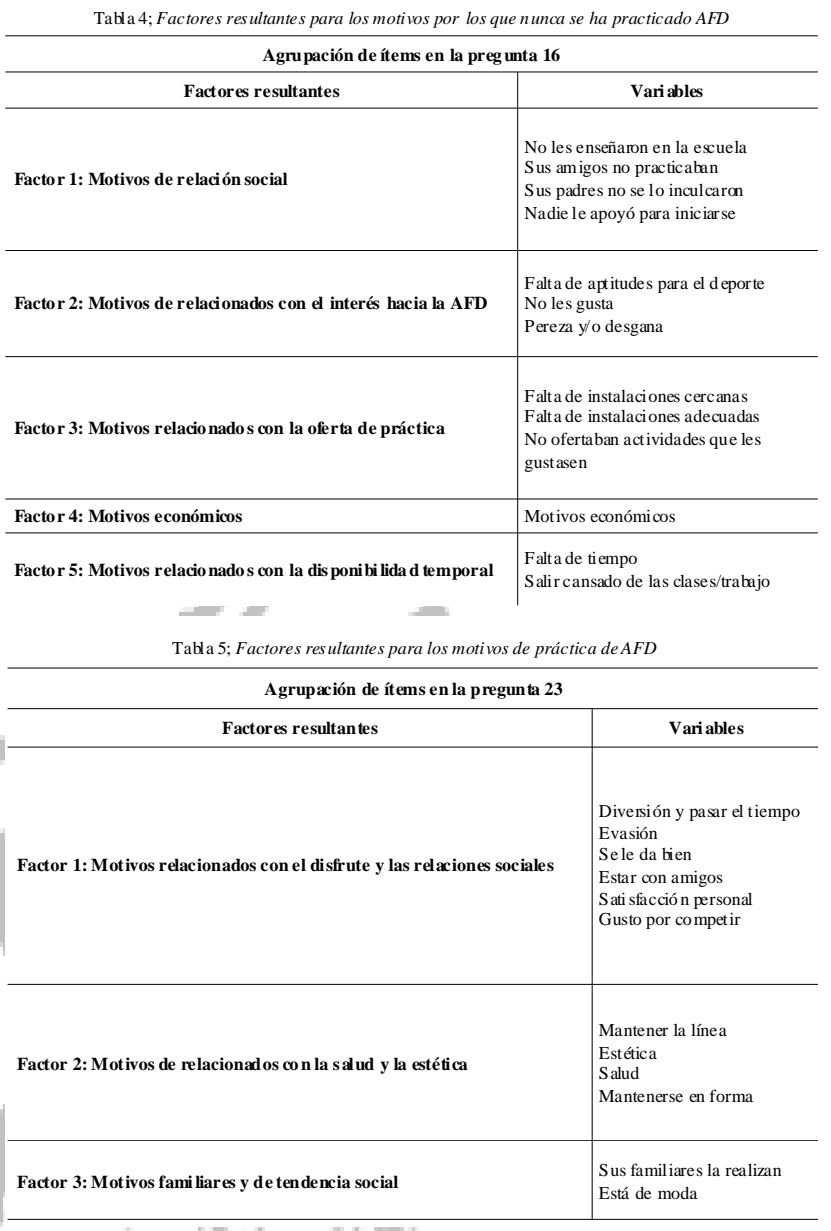

dando finalmente las variables agrupadas en las siguientes dimensiones, tal como puede observarse en las siguientes tablas.

El cuestionario fue administrado durante el último cuatrimestre del curso 2009/2010 en los grupos seleccionados aleatoriamente durante el horario de clases. Todos los sujetos encuestados accedieron voluntariamente a participar tras recibir las instrucciones de los investigadores. Durante la recogida de datos siempre hubo un investigador presente.

Se ha realizado un análisis descriptivo de los datos a través del programa estadístico SPSS versión 15.0.

\section{Resultados}

Como se observa en la figura 1 , las principales razones aducidas por el alumnado activo (62.19\%) de esta Facultad para practicar AFD son, en primer lugar, el mantenerse en forma, con una puntuación media de 4.35 , seguido de cerca por la salud (4.26) y la satisfacción personal (4.08). El mantenimiento de la línea (3.89), la diversión y el pasatiempo (3.88), la evasión (3.5) y la estética también son motivos valorados por los estudiantes.

El 10.48\% de los estudiantes que no han practicado nunca AFD señala como razones más relevantes para ello la falta de tiempo (3.90), el salir cansado de las clases y/o el trabajo (3.30) y la pereza y desgana (3.02), seguidos de la falta de gusto por el deporte (2.69) o el que sus amigos no practicasen (2.37), entre otras.

Entre los motivos más relevantes que aduce el alumnado (27.56\%) para haber abandonado la práctica de AFD encontramos, en primer lugar y con una puntuación media bastante aventajada sobre el resto (4.01), la falta de tiempo. A continuación se destacan otros motivos como el salir cansado de las clases y/o el trabajo (3.22), la falta de instalaciones deportivas cercanas (2.75), la pereza y desgana (2.49), entre otras. De estos alumnos que dejaron de realizar AFD, más del $80 \%$ de los mismos estaría interesado en retomar la práctica y entre los factores que les ayudarían a encontramos, entre los más valorados, la 
organización del tiempo de estudio (4.28) y la compatibilidad de la práctica con el horario de clases (4.20), así como que las actividades fuese más económicas (4.14) y dispusieran de instalaciones cercanas para la práctica (4.09).

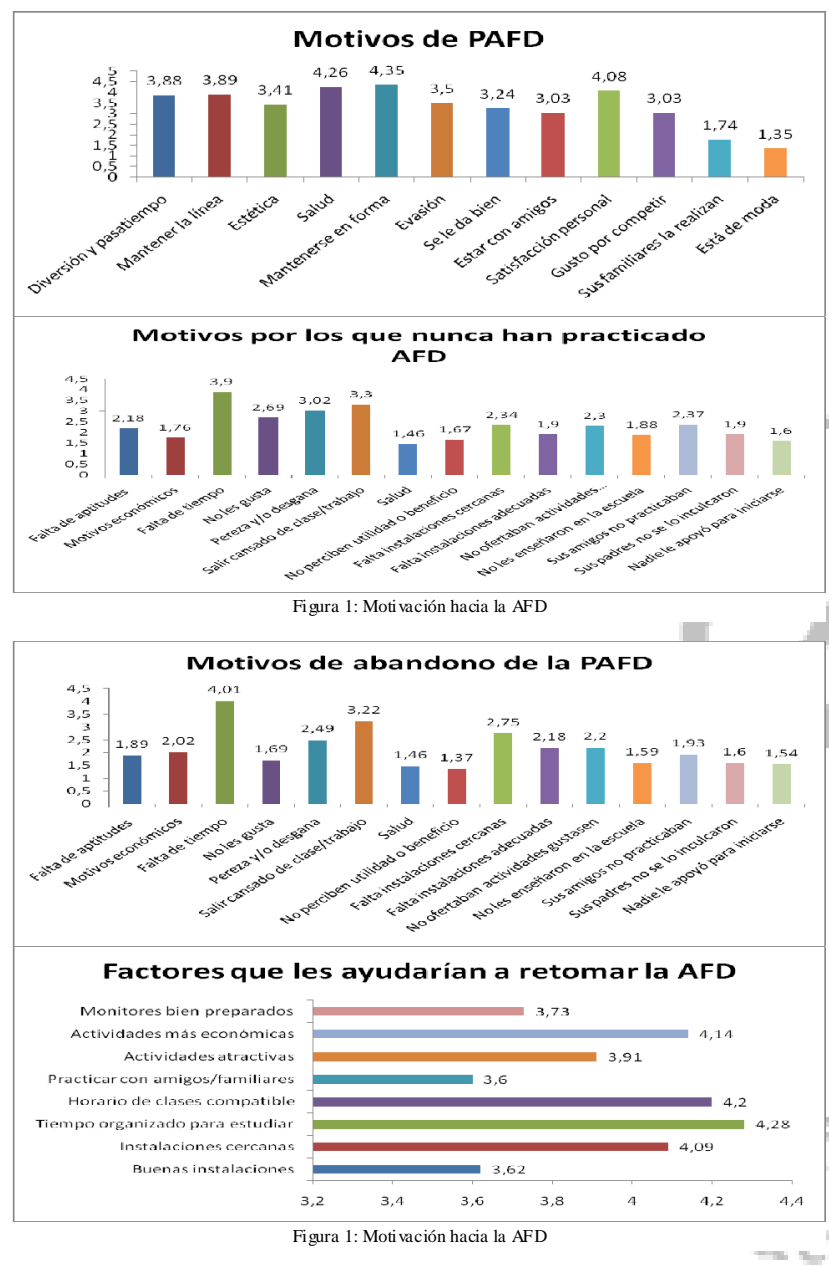

\section{Análisis y discusión de los resultados}

Los estudiantes de la Facultad de Ciencias de la Educación, a la hora de practicar $\mathrm{AFD}$, valoran más los motivos relacionados con la salud y la estética, así como los relacionados con el disfrute y las relaciones sociales. Si comparamos estas motivaciones con las obtenidas por otros investigadores en otras universidades, observamos que hay varios motivos que aparecen como una constante entre los más valorados por los estudiantes universitarios. En la siguiente tabla, pueden observarse los principales motivos de prácticas del alumnado de diversas universidades.

\begin{tabular}{ccccccc}
\multicolumn{7}{c}{ Tabla 6; Motivos por los que los estudiantes universitarios practican AFD } \\
\hline Motivaciones & $\mathbf{1}$ & $\mathbf{2}$ & $\mathbf{3}$ & $\mathbf{4}$ & $\mathbf{5}$ & $\mathbf{6}$ \\
\hline $\begin{array}{c}\text { Universid ad de } \\
\text { Guad alajara }\end{array}$ & $\begin{array}{c}\text { Mejorar la } \\
\text { salud }\end{array}$ & $\begin{array}{c}\text { Mejorar la } \\
\text { forma física }\end{array}$ & Diversión & $\begin{array}{c}\text { Hacer } \\
\text { ejercicio }\end{array}$ & $\begin{array}{c}\text { Mejorar las } \\
\text { habilidades } \\
\text { personales }\end{array}$ & Rel ajación \\
\hline $\begin{array}{c}\text { Universidad de } \\
\text { La Rioja }\end{array}$ & Diversión & Me gu sta & $\begin{array}{c}\text { Mejojar la } \\
\text { forma física }\end{array}$ & $\begin{array}{c}\text { Gusto por el } \\
\text { ejercicio }\end{array}$ & Relajación & $\begin{array}{c}\text { Estar con } \\
\text { amigos }\end{array}$ \\
\hline $\begin{array}{c}\text { Universidad de } \\
\text { Huelva }\end{array}$ & $\begin{array}{c}\text { Hacer } \\
\text { ejercicio }\end{array}$ & $\begin{array}{c}\text { Mejorar la } \\
\text { salud }\end{array}$ & Me gusta & $\begin{array}{c}\text { Diversión y } \\
\text { pasatiempo }\end{array}$ & Satisfacción & $\begin{array}{c}\text { Mantener la } \\
\text { línea }\end{array}$ \\
\hline $\begin{array}{c}\text { Universidad de } \\
\text { Sevilla }\end{array}$ & $\begin{array}{c}\text { Estar en } \\
\text { forma }\end{array}$ & $\begin{array}{c}\text { Diversión y } \\
\text { pasatiempo }\end{array}$ & $\begin{array}{c}\text { Hacer } \\
\text { ejercicio }\end{array}$ & $\begin{array}{c}\text { Mejorar la } \\
\text { salu d }\end{array}$ & Evadirse & $\begin{array}{c}\text { Estar con } \\
\text { amigos }\end{array}$ \\
\hline $\begin{array}{c}\text { Facultad de } \\
\text { CC. de la }\end{array}$ & $\begin{array}{c}\text { Estar en } \\
\text { forma }\end{array}$ & $\begin{array}{c}\text { Sati sfacción } \\
\text { personal }\end{array}$ & $\begin{array}{c}\text { Mantener la la } \\
\text { línea }\end{array}$ & $\begin{array}{c}\text { Diversión y } \\
\text { pasatiempo }\end{array}$ & Evadirse & Estética \\
\hline
\end{tabular}

Uni versidad de Guadalajara (Flores, 2009); Uni versidad de La Rioja (Sanz, 2005); Univ ersidad de Huelva (Castillo \& Sáenz-López, 2008); Universidad de Sevilla (Rodríguez \& Sánchez, 2010)
De este modo, se observa que la diversión y el pasatiempo aparecen como uno de los motivos más valorados en todos los estudios contemplados. En segundo lugar, el estar en forma aparece en cuatro de las cinco investigaciones, seguido de la mejora y/o mantenimiento de la salud y el hacer ejercicio, que aparecen en tres de los cinco estudios, aunque ninguno de ellos forma parte de los motivos más valorados por los estudiantes de la Facultad de Ciencias de la Educación. Otros motivos como la evasión, la relajación, el mantenimiento o el cuidado de la línea y la estética son también parte de esta constante de motivaciones para la práctica de los universitarios.

Todos los datos coinciden con otros estudios sobre motivaciones para la práctica de AFD en alumnado universitario, en los que se observa que las motivaciones más importantes para la práctica por parte de estos sujetos son de carácter intrínseco, primando la mejora de la salud y la forma física, el bienestar, la diversión, la evasión y otras motivaciones alejadas de los resultados de la competición y otros factores extrínsecos de la práctica (Torres, Carrasco \& Medina, 2000; Gómez, Ruiz, García, Granero \& Pieron, 2009).

Por otro lado, Moreno, Pavón, Gutiérrez y Sicilia (2005), en una investigación realizada en las Universidades de Murcia, Valenciay Almería, observaron diferencias en las motivaciones hacia la práctica de AFD por parte del alumnado practicante y no practicante. Así, aquellos que realizaban AFD encontraban mayor motivación en la competición, en ser eficaces y en el mantenimiento de la forma física que los que no practicaban, quienes se decantaban por motivos relacionados con la salud.

De esta forma, podemos considerar que las motivaciones del alumnado practicante de la Facultad de Ciencias de la Educación son bastante similares a las del alumnado de otras universidades, y coincidimos con Dosil (2004) en que no existe un único motivo que impulse a los estudiantes universitarios a mantener un comportamiento activo, sino un conjunto de ellos, de manera que podríamos incluso advertir la existencia de unas motivaciones comunes a este grupo de población concreto.

En cuanto a las motivaciones aducidas por el alumnado para no haber practicado nunca $\mathrm{AFD}$, se observan de nuevo coincidencias entre los resultados de presente investigación y los obtenidos en otros estudios sobre universitarios. En la siguiente tabla puede observarse que la falta de tiempo aparece valorado en las cuatro investigaciones contempladas, y en tres de ellas, como el principal motivo por el que el alumnado no ha practicado nunca AFD. La flojera y/o pereza también aparece en todas las investigaciones como otro de los motivos más valorados, asî como la falta de instalaciones, ya sean cercanas o adecuadas, se contempla en todos los estudios excepto en el de Sanz (2005). De este modo encontramos de nuevo una serie de motivaciones comunes en los universitarios por las que no han practicado nunca $\mathrm{AFD}$, pudiendo considerar como las más importantes la falta de tiempo y la pereza o desgana.

Atendiendo al abandono de la práctica de $\mathrm{AFD}$, la falta de tiempo aparece de nuevo como motivo más valorado por los estudiantes de la Facultad de Ciencias de la Educación. Los motivos más importantes

\begin{tabular}{|c|c|c|c|c|c|c|}
\hline Motivaciones & 1 & 2 & 3 & 4 & 5 & 6 \\
\hline $\begin{array}{c}\text { Universidad } \\
\text { de } \\
\text { Guadalajara }\end{array}$ & $\begin{array}{c}\text { Falta de } \\
\text { aptitudes } \\
\text { para el } \\
\text { deporte }\end{array}$ & $\begin{array}{c}\text { Flojera o } \\
\text { pereza }\end{array}$ & $\begin{array}{l}\text { Falta de } \\
\text { tiempo }\end{array}$ & $\begin{array}{c}\text { Falta de } \\
\text { instalaciones } \\
\text { cercanas }\end{array}$ & $\begin{array}{l}\text { Nole gusta } \\
\text { el deporte }\end{array}$ & $\begin{array}{l}\text { Sus padres } \\
\text { no se lo } \\
\text { inculcaron }\end{array}$ \\
\hline $\begin{array}{l}\text { Universidad } \\
\text { de La Rioja }\end{array}$ & $\begin{array}{l}\text { Falta de } \\
\text { tiempo }\end{array}$ & Pereza & $\begin{array}{c}\text { Salir cansado } \\
\text { de estudios o } \\
\text { trabajo }\end{array}$ & $\begin{array}{c}\text { Preferencia } \\
\text { de otras } \\
\text { actividades } \\
\text { de tiempo } \\
\text { libre }\end{array}$ & $\begin{array}{l}\text { Falta de } \\
\text { interés }\end{array}$ & Estudi os \\
\hline $\begin{array}{l}\text { Universidad } \\
\text { de Huelva }\end{array}$ & $\begin{array}{l}\text { Falta de } \\
\text { tiempo }\end{array}$ & $\begin{array}{l}\text { Perezao } \\
\text { desgana }\end{array}$ & $\begin{array}{l}\text { Mo tivos } \\
\text { económicos }\end{array}$ & $\begin{array}{c}\text { Falta de } \\
\text { instalaciones } \\
\text { adecuadas }\end{array}$ & $\begin{array}{c}\text { Sus amigos } \\
\text { no } \\
\text { practicaban }\end{array}$ & $\begin{array}{c}\text { No se le da } \\
\text { bien el } \\
\text { deporte }\end{array}$ \\
\hline $\begin{array}{l}\text { Facultad de } \\
\text { CC. de la } \\
\text { Educación }\end{array}$ & $\begin{array}{l}\text { Falta de } \\
\text { tiempo }\end{array}$ & $\begin{array}{c}\text { Salir } \\
\text { cansado de } \\
\text { clase o del } \\
\text { trabajo }\end{array}$ & $\begin{array}{l}\text { Pereza o } \\
\text { desgana }\end{array}$ & $\begin{array}{l}\text { Falta de } \\
\text { gusto por el } \\
\text { departe }\end{array}$ & $\begin{array}{l}\text { Sus amigos } \\
\text { no } \\
\text { practicaban }\end{array}$ & $\begin{array}{c}\text { Falt a de } \\
\text { instalaciones } \\
\text { cercanas }\end{array}$ \\
\hline
\end{tabular}

Uni versidad de Guadalajara (Flores, 2009); Uni versidad de La Rioja (Sanz, 2005); Univ ersidad de Huelva (Castillo \& Sáenz-López, 2008); Universidad de Se villa (Rodríguez \& Sánchez, 2010) 
para este grupo de alumnos están relacionados con la disponibilidad temporal, la oferta de práctica y el interés hacia la AFD. Así, si volvemos a comparar las motivaciones con las obtenidas por otros investigadores en otras universidades, observamos que se repiten algunas de manera constante entre diferentes grupos de estudiantes. En la tabla 8 se exponen los principales motivos de abandono del alumnado de diversas universidades y se observa que el motivo más señalado por los estudiantes universitarios para haber abandonado la AFD es la falta de tiempo, presente en cuatro de las cinco investigaciones contempladas. Le siguen la pereza o desgana, la falta de instalaciones cercanas, la incompatibilidad con otras obligaciones y los problemas de salud y/o lesiones, todos ellos presentes en tres de los cinco estudios.

\begin{tabular}{|c|c|c|c|c|c|c|}
\hline Motivaciones & 1 & 2 & 3 & 4 & 5 & 6 \\
\hline $\begin{array}{c}\text { Universidad } \\
\text { de } \\
\text { Guadalajara }\end{array}$ & $\begin{array}{c}\text { Incompatibil i- } \\
\text { dad con } \\
\text { ob ligaciones } \\
\text { académicas } \\
\text { y/o laborales }\end{array}$ & $\begin{array}{c}\text { Flojera o } \\
\text { pereza }\end{array}$ & $\begin{array}{c}\text { Falta de } \\
\text { instal acione } \\
\text { s cercanas }\end{array}$ & $\begin{array}{l}\text { Cambio de } \\
\text { centro escolar }\end{array}$ & $\begin{array}{c}\text { Falta de } \\
\text { compañía } \\
\text { con quien } \\
\text { practicar }\end{array}$ & $\begin{array}{c}\text { Problemas de } \\
\text { salud o } \\
\text { lesiones }\end{array}$ \\
\hline $\begin{array}{l}\text { Universidad } \\
\text { de La Rioja }\end{array}$ & $\begin{array}{l}\text { Falta de } \\
\text { tiempo }\end{array}$ & $\begin{array}{l}\text { Por los } \\
\text { estudios }\end{array}$ & Pereza & $\begin{array}{l}\text { Incompat ibili- } \\
\text { dad con } \\
\text { obl igaciones }\end{array}$ & $\begin{array}{c}\text { Otras } \\
\text { preferencias } \\
\text { para el } \\
\text { ti empo li bre }\end{array}$ & $\begin{array}{l}\text { Falt ade } \\
\text { interés }\end{array}$ \\
\hline $\begin{array}{l}\text { Universidad } \\
\text { de } \mathrm{H} \text { uelva }\end{array}$ & $\begin{array}{l}\text { Falta de } \\
\text { tiempo para } \\
\text { estudiar }\end{array}$ & $\begin{array}{l}\text { Cambio de } \\
\text { centro de } \\
\text { estudios }\end{array}$ & $\begin{array}{l}\text { Abandono } \\
\text { de amigos }\end{array}$ & Lesiones & Trabajo & $\begin{array}{c}\text { Exigencias } \\
\text { del } \\
\text { entrenador }\end{array}$ \\
\hline $\begin{array}{c}\text { Universidad } \\
\text { de Sevilla }\end{array}$ & $\begin{array}{l}\text { Falta de hábito } \\
\text { de práctica }\end{array}$ & $\begin{array}{l}\text { Falta de } \\
\text { tiempo }\end{array}$ & $\begin{array}{c}\text { Salir } \\
\text { cansado de } \\
\text { estudios y/o } \\
\text { trabajo }\end{array}$ & $\begin{array}{c}\text { Horari os } \\
\text { incompat ibles }\end{array}$ & Lesiones & $\begin{array}{c}\text { Falt ade } \\
\text { instalaciones } \\
\text { cercanas }\end{array}$ \\
\hline $\begin{array}{l}\text { Facultad de } \\
\text { CC.dela } \\
\text { Educación }\end{array}$ & $\begin{array}{l}\text { Falta de } \\
\text { tiempo }\end{array}$ & $\begin{array}{c}\text { Salir } \\
\text { can sado } \\
\text { de clase } \\
\text { y/o trabajo }\end{array}$ & $\begin{array}{l}\text { Falta de } \\
\text { instal acione } \\
\text { s cercanas }\end{array}$ & $\begin{array}{l}\text { Pereza o } \\
\text { desgana }\end{array}$ & $\begin{array}{c}\text { Falta de } \\
\text { acti vida-des } \\
\text { atrac-tivas }\end{array}$ & $\begin{array}{c}\text { Falt a de } \\
\text { instalaciones } \\
\text { adecuadas }\end{array}$ \\
\hline
\end{tabular}

Estos datos también coinciden con los de Cecchini y González (2008), que obtuvieron como principales motivos del alumnado de la Universidad de Oviedo para haber dejado de practicar AFD, la falta de tiempo, la pereza y la falta de instalaciones. Según estos autores, estas motivaciones de carácter extrínseco se encuentran directamente relacionadas con la gestión del deporte universitario, por lo que una buena política y gestión por parte de la institución podría paliar de forma importante este problema.

Podemos pensar entonces, que los motivos de abandono del alumnado de la Facultad de Ciencias de la Educación son bastante similares a los del alumnado de otras universidades. Y podríamos advertir también, la existencia de algunas motivaciones principalmente de carácter extrínseco, comunes a este grupo de población y paliables en cierta medida a través de mejoras en la gestión deportiva.

Así mismo, si comparamos los resultados de la tabla 8, sobre los motivos de abandono de la práctica de AFD y los de la tabla 7, sobre los motivos por los que nunca han realizado AFD, comprobamos que las motivaciones que llevan al alumnado a abandonar la práctica y las que le han llevado a no haber practicado nunca pueden considerarse muy similares, coincidiendo principalmente en la falta de tiempo, el salir cansado de clase o del trabajo y la pereza o la desgana. Además, como señalan Ruiz y Gómez (2005), el hecho de que exista una mayoría de estudiantes universitarios que señalan la falta de tiempo entre los motivos más importantes para no haber realizado nunca AFD o haber dejado de practicarla, supone que la oferta deportiva no está teniendo en cuenta las características y necesidades de este colectivo para intentar frenar el abandono deportivo y captar nuevos practicantes.

En cuanto a la disposición para retomar la práctica de AFD, se observa que más del $80 \%$ del alumnado de la Facultad de Ciencias de la Educación estaría dispuesto a volver a realizar AFD, y entre los factores que más les ayudarían a ello encontramos aquellos que están relacionados precisamente con la organización y la disponibilidad temporal, como el tener el tiempo organizado para estudiar y que el horario de clases fuese compatible con las actividades. Esto concuerda con los datos obtenidos por Castillo y Sáenz-López (2008) en Huelva, donde los universitarios señalaban también el tener organizado el tiempo para estudiar en primer lugar, seguido de cerca por tener un horario de actividades compatible con el de clase, tener una oferta de actividades atractivas y que el monitor estuviese bien preparado, entre otros. De este modo, podemos considerar que una buena disposición y organización temporal es valorado como uno de los motivos más importantes para retomar la práctica, lo que concuerda con esa falta de tiempo que señalaban los estudiantes, tanto los que habían abandonado la práctica como los que no la habían realizado nunca, así como nos hace volver a considerar, tal y como señalaban Ruiz y Gómez (2005), si verdaderamente la oferta deportiva universitaria está teniendo en cuenta las características y necesidades de sus estudiantes, tanto para poner fin al abandono como para favorecer la captación de nuevos practicantes.

\section{Conclusiones}

Los principales motivos por los que los estudiantes de la Facultad de Ciencias de la Educación realizan AFD están relacionados principalmente con la mejora de la salud y la estética, así como con el disfrute y las relaciones sociales, quedando en un segundo lugar aquellas motivaciones relacionadas directamente con la competición y los éxitos deportivos.

En cuanto a los motivos aducidos por el alumnado para no haber practicado nunca AFD o haber dejado de hacerlo, son muy semejantes en ambos casos y se centran en la disponibilidad temporal, el interés hacia la AFD y la oferta de práctica. La mayoría del alumnado que abandonó la AFD estaría dispuesto a retomar la práctica, señalando como los factores que más le ayudarían a ello aspectos de organización y disposición temporal, tales como tener bien organizado su tiempo de estudio o disponer de actividades compatibles con su horario de clase.

En función de estos resultados y de su comparativa con otros obtenidos en diferentes investigaciones sobre estudiantes universitarios, cabe plantearse si realmente las instituciones tienen en cuenta los gustos y las necesidades de los estudiantes en relación a la práctica de $\mathrm{AFD}$, así como si estas motivaciones extrínsecas del alumnado que dificultan la práctica podrían modificarse a través de una buena gestión deportiva por parte de la institución universitaria.

\section{Gitiving}

\section{Referencias}

Castillo, E., \& Sáenz-López, P. (2008). Práctica de actividad física y estilo de vida del alumnado de la Universidad de Huelva. España: Servicio de Publicaciones de la Universidad de Huelva.

Cecchini, J. A., \& González, C. (2008). Motivos por los que los estudiantes universitarios no practican deporte. Revista Fuentes, 8 , 199-208.

Consejo Superior de Deportes-CSD, (2006). El modelo del Deporte Universitario Español. Córdoba: Universidad de Córdoba.

Consejo Superior de Deportes-CSD, (2010). Encuesta sobre los hábitos deportivos en España 2010. Avance de resultados. Recuperado de http://www.csd.gob.es/csd/estaticos/noticias/DOSSIERENCUESTA.pdf

Darren, E. R., Crystal, N., \& Shannon, B. (2006). Health benefits of physical activity: the evidence. Canadian Medical Association Journal, 174(6), 801-809.

Dosil, J. (2004). Psicología de la actividad física y del deporte. Madrid: McGraw-Hill.

Escartí, A., \& Cervelló, E. (1994). La motivación en el deporte. En I. Balaguer(Ed.), Entrenamiento psicológico en deporte: Principios y aplicaciones (pp. 61-90). Valencia: Albatros Educación.

Ferrando, P. F., \& Anguiano-Carrasco, C. (2010). El análisis factorial como técnica de investigación en psicología. Papeles del Psicólogo, $31(1), 18-33$.

Flores, G (2009). Actividad físico-deportiva del alumnado de la Universidad de Guadalajara (México). Correlatos biológicos y cognitivos asociados. Tesis Doctoral. Universidad de San Javier (Murcia). 
García, M. (2001). Los españoles y el deporte. Prácticas y comportamientos de la última década del siglo XX. Encuesta sobre los hábitos deportivos de los españoles, 2000. Madrid: Ministerio de Educación y Cultura. Consejo Superior de Deportes.

García, M. (2006). Postmodernidad y Deporte: Entre la individualización y la masificación. Encuesta sobre los hábitos deportivos de los españoles 2005. Madrid: CSD/CIS.

Gómez, M., Ruiz, F., García, M.E., Granero, A., \& Piéron, M. (2009). Motivaciones aludidas por los universitarios que practican actividades físico-deportivas. Revista Latinoamericana de Psicología, 41(3), 519-532.

Ley Orgánica 6/2001, de 21 de diciembre, de Universidades. Recuperado de http://www.boe.es/boe/dias/2001/12/24/pdfs/A4940049425.pdf.

Ley Orgánica 4/2007, de 12 de abril, de Universidades. Recuperado de http://www.boe.es/boe/dias/2007/04/13/pdfs/A16241-16260.pdf

López, A., \& González, V. (2001). Niveles de satisfacción por la clase de Educación Física. Lecturas: Educación Física y Deporte, Revista Digital, 32. Recuperado de http//www.efdeportes.com/efd32/ satisf.htm

Lowenthal, K. M. (2001). An introduction to psychological tests and scales. Hove and New York: Psychology Press.

Martínez-López, E. (2003). La salud como motivación para la práctica de actividad física en personas adultas. Iatreia, 16(1), 32-43.

McMillan, J. H. (2008). Assessment Essentials for Standards-Based Education. New York: Corwin Press.

Moreno, J. A., Cervelló, E., \& Martínez Camacho, A. (2007b). Validación de la Escala de Medida de los Motivos para la Actividad Física-Revisada en españoles: Diferencias por motivos de participación. Anales de Psicología, 23, 167-176.

Moreno, J. A.; Pavón, A. I., Gutiérrez, M., \& Sicilia, A. (2005). Motivaciones de los universitarios hacia la práctica físico-deportiva.
Revista Internacional de Medicina y Ciencias de la Actividad Física y el Deporte, 5(19), 154-165.

Palou, P., Ponseti, X., \& Borrás, P. A. (2001). Hábitos deportivos de los estudiantes de la universidad de las Islas Baleares. Educación y Cultura, 14, 163-180.

Pavón, A., \& Moreno, J. A. (2006). Opinión De los universitarios sobre la EF y el deporte. Revista de Ciencias de la Actividad Física, $U C M, 8(8), 25-34$

Pìeron, M., Ruíz, J., \& García, M. A. (2009). Actividad física y estilos de vida saludables. Wanceulen: Sevilla.

Rodríguez, A., \& Sánchez, E. (2010). Los universitarios y el deporte. Sevilla: Wanceulen.

Ruiz, F., García, M. E., \& Pieron, M. (2009). Actividad física y estilos de vida saludables. Análisis de los determinantes de la práctica en adultos. Sevilla: Wanceulen.

Ruiz, F., \& Gómez, M. (2005). Práctica deportiva en los universitarios. En M.L. Zagalaz, E.J. Martínez y P.A. Latorre, Respuestas a la demanda social de actividad física (pp. 143-160). España: Gymnos.

Sánchez-Barrera, M.; Pérez, M., \& Godoy, J. (1995). Patrones de actividad física de una muestra española. Revista de Psicología del Deporte, 7-8, 51-71.

Sanz, E. (2005). La práctica físico-deportiva de tiempo libre en universitarios. Análisis y propuesta de mejora. Logroño: Servicio de $\mathrm{Pu}-$ blicaciones de la Universidad de La Rioja.

Torres, G, Carrasco, L., \& Medina, J. (2000). Investigación sobre los motivos por los que los estudiantes universitarios practican deporte. El caso de la Universidad de Granada. Motricidad, 6, 95-106.

Trost, S. G., Kerr, L. M., Ward, D. S., \& Pate, R. R. (2001). Physical activity and determinants of physical activity in obese and nonobese children. International Journal of Obesity, 25, 812-829.

Van der Host, K., Paw, M. S., Twisk, J. W., \& Van Mechelen, W. (2007). A Brief Review on Correlates of Physical Activity and Sedentariness in Youth. Medicine and Science in Sporte Exercise, 39(8), 1241-1250.

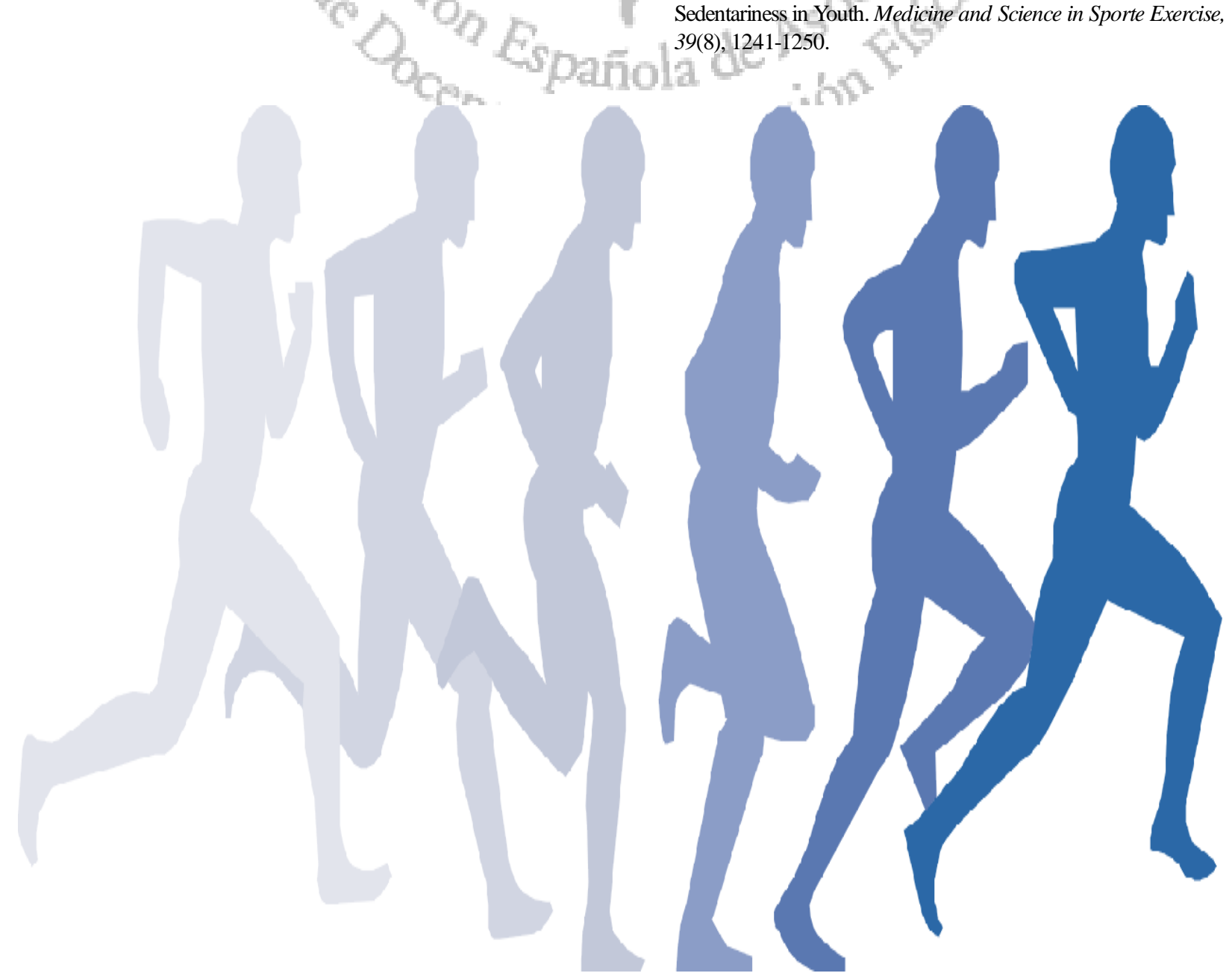

